\title{
Psilocybin Combines Rapid Synaptogenic And Anti- Inflammatory Effects In Vitro
}

Gabriella Smedfors ( $\square$ gabriella.smedfors@ki.se )

Karolinska Institute

\section{Elliot Glotfelty}

National Institute on Aging

Negar Kalani

Karolinska Institute

Christian Papatziamos Hjelle

Karolinska Institute

Otilia Horntvedt

Karolinska Institute

Katrin Wellfelt

Karolinska Institute

Alvin Brodin

Karolinska Institute

Fredrik von Kieseritzky

Karolinska Institute

Lars Olson

Karolinska Institute

Tobias Karlsson

Karolinska Institute

\section{Research Article}

Keywords: psilocybin, psychedelics, synaptic plasticity, neuroinflammation, microglia, neurons

Posted Date: March 8th, 2022

DOI: https://doi.org/10.21203/rs.3.rs-1321542/v1

License: (1) This work is licensed under a Creative Commons Attribution 4.0 International License. Read Full License 


\section{Abstract}

Psilocybin is a psychedelic substance approaching clinical use. The drug has long-lasting effects after single or multiple administrations and enhances structural plasticity in the brain. Little is known if the plasticity inducing effects of psilocybin could be timed to other treatments and promote a larger effect. We investigated the effect of psilocybin on cultured mouse hippocampal neurons, examining the plasticity promoting effects from $5 \mathrm{~min}$ to $72 \mathrm{~h}$ post-treatment. We found robust effects on pre- and postsynaptic (Piccolo and Homer1) protein expression 1-3 h following treatment. Presynaptic Synapsin-1 expression mirrored these findings, with peak expression $72 \mathrm{~h}$ post-treatment. Our studies suggest psilocybin opens a window of plasticity that rapidly normalizes. As psilocybin has been shown to have an effect treating diseases (e.g. depression and cluster headache) linked with inflammation, we used an immortalized microglia cell line (IMG) to demonstrate its anti-inflammatory effects against a lipopolysaccharide (LPS) challenge (we show reduced tumor necrosis factor-alpha (TNF-a) secretion). Altogether, our studies show discrete and acute cell type specific effects of psilocybin that provides insight into its mechanisms of action and potential therapeutic value.

\section{Introduction}

The central nervous system is a growth inhibitory environment ${ }^{1}$. This is not due to an intrinsic lack of capacity of neurites and synapses to undergo changes, but to the default presence of components that limit their ability to do so, including the Nogo signaling system ${ }^{2,3}$. A growth inhibitory environment is beneficial for maintaining stable neural connections, however, in cases of pathological states or injury, the inability for neurons to grow or repair can be a detrimental ${ }^{4}$. Endogenous opposition to this restrictive growth environment comes from neurotrophins, such as brain-derived neurotrophic factor (BDNF) ${ }^{5}$ and glial cell line derived neurotrophic factor (GDNF) ${ }^{6}$, both of which exert positive effects on structural plasticity. Interest in compounds or procedures that may have similar effects remains a hallmark of historical and modern neuroscience research 7,8 .

Recently, interest into psychedelic drugs has led to a burgeoning body of work demonstrating the effects of these once stigmatized substances, a number of which were recently shown to induce neurite outgrowth as well as spine sprouting ${ }^{9}$. One of these substances was psilocin, the biologically active metabolite of psilocybin ${ }^{10}$. Psilocybin is rapidly dephosphorylated to psilocin, an unspecific serotonin agonist ${ }^{11,12}$, where signaling through the 5-hydroxytryptamine (5-HT) $2 \mathrm{~A}$ receptor is believed to be responsible for hallucinogenesis ${ }^{13}$. Psilocybin has, in line with the other psychedelic substances, a favorable safety profile, including non-addictive properties and a large margin of exposure, compared to most central nervous system (CNS)-active drugs ${ }^{13-16}$.

Psilocybin is currently being evaluated for its role in the treatment of several CNS disorders, and evidence is accumulating in its favor. For example, a recent phase 2 clinical trial illustrated therapeutic potential against major depressive disorder ${ }^{17}$, and addiction has previously been targeted with remarkable 
effect ${ }^{18-20}$. Additionally, retrospective positive findings ${ }^{21,22}$ have indicated potential therapeutic value of psilocybin against cluster headache. Clinical trials (NCT04280055 and NCT02981173) are currently recruiting for these patients, who have little reprieve from the debilitating disorder. Evidence of prolonged migraine suppressing effects of a single dose of psilocybin were recently demonstrated ${ }^{23}$, further supporting the use of psilocybin analogues for treatment of headache disorders. Despite growing evidence for efficacy across a variety of maladies, little is known about the mechanistic effects of psilocybin and its long-lasting effects after single or multiple administrations ${ }^{24-27}$.

Psilocybin has previously been shown to have a dose-dependent effect on hippocampal neurogenesis ${ }^{28}$. It is also important to investigate potential plasticity enhancing properties at the synaptic level. There is also growing evidence of an anti-inflammatory capacity of serotonergic system affecting compounds, including psilocybin ${ }^{29,30}$. Microglial cells are the resident macrophages and innate immune cells of the brain and respond to damage or aberrant neuronal signaling with, among other roles, rapid secretion of proteins involved in inflammation, including cytokines, chemokines, and other factors ${ }^{31}$. A recent study using mouse macrophages demonstrated reductions of canonical inflammatory markers, including TNFa, with extracts of the mushroom Psilocybe Natalensis ${ }^{32}$. As neuroinflammation is a common factor across neuropsychiatric disorders ${ }^{33}$, as well as major depressive disorder ${ }^{34}$, it represents another target to which psilocybin may exert effects on the brain.

Here, we provide evidence regarding acute and lasting effects of psilocybin on synaptic and structural plasticity. We demonstrate that cultured hippocampal neurons respond rapidly to psilocybin, increasing the number of synaptic puncta detected with antibodies against three different synaptic proteins over a course of 72 hours after treatment. Cultured cortical neurons responded opposite to hippocampal cultures, indicating possible region-specific effects of the drug. Overall, however, our results support prior research indicating psilocybin's potent impact on synaptic plasticity. For a more holistic perspective on cell type specific interactions in the brain, we used an immortalized microglial cell (IMG) line to demonstrate significant anti-inflammatory effects of psilocybin against an LPS challenge, epitomized by decreases in TNF-a secretion.

\section{Material And Methods}

\subsection{Animals}

C57BL/ 6 mice (Janvier Labs, France) were housed in the KMB animal department at Karolinska Institutet in shared ventilated boxes (2-3 mice per box) with food and water ad libitum. Lights were on a $12 \mathrm{~h}$ lightdark cycle, temperature was kept at $22-23^{\circ} \mathrm{C}$, and relative humidity was set to $60 \%$. A paper house and tissues were provided for nesting. All animal experiments were approved by the Northern Stockholm Animal Ethical Committee (Stockholms Norra Djurförsöksetiska nämnd), permit number N246/15, Permit in line with EU Directive 2010/63/EU for animal experiments. The study is reported in accordance with ARRIVE guidelines. 


\subsection{Psilocybin vs psilocin}

When psilocybin is metabolized in vivo, it undergoes rapid dephosphorylation into its CNS-active form psilocin (Fig. 1) ${ }^{35}$. Psilocybin should thus be considered a prodrug for psilocin. Previous in vitro and in vivo work investigating effects of psilocybin on behavior and neuronal plasticity has often been carried out directly with psilocin $9,36,37$. However, psilocin is notoriously unstable, as it is a hydroxy-indole, making it difficult to store and handle in a way that avoids auto-degradation ${ }^{38}$. Meanwhile, as the abundance of relevant phosphatases such as tissue-nonspecific alkaline phosphatases found in representative glial cell and neuronal cultures ${ }^{39}$, facilitates psilocybin to be effectively converted to psilocin in vitro ${ }^{40,41}$, we chose to use psilocybin (Merck \& Co, USA cat\#P-097-1ML) for our studies. Observed effects should thus be attributed to psilocin.

\subsection{Cell cultures2.3.1 Primary Neurons:}

Cell cultures were derived from dissected hippocampal tissue of E18 C57BL6 mice (Janvier labs). Cells were dissociated by incubating the fetal brain tissue in trypsin in a $37^{\circ} \mathrm{C}$ water bath for $15 \mathrm{~min}$. Tubes were turned every 3rd min. The trypsinized tissue was washed three times in HBSS and subsequently separated with normal and fire polished glass Pasteur pipettes. Cells were stained with Trypan blue, counted in a Bürker chamber and plated on polyornithine coated coverslips in 24-well plates at densities of 37,500 cells per well for hippocampal cultures and 75,000 cells per well for cortical neurons. The media differed slightly: for hippocampal cells we used an attachment media consisting of Neurobasal media, $200 \mathrm{mM}$ glutamine and B27 supplement serum; for cortical cells we used Neurobasal + and B270+ (all reagents from Thermo Fisher Scientific). Cell cultures were incubated $\left(37^{\circ} \mathrm{C}, 5 \% \mathrm{CO}^{2}\right)$, and three hours after plating, the attachment medium was changed to maintenance medium (attachment media with $1 \%$ penicillin and streptomycin). At day in vitro (DIV) 17, cultures were analyzed for synapse markers.

\subsubsection{Immortalized Microglia (IMG) Cells:}

The immortalized mouse microglia ${ }^{43}$ (Sigma-Aldrich, cat\# SCC-134) cell line was used to model neuroinflammation. Cells were cultured in tissue culture treated 24 well plates in High Glucose Dulbecco's Modified Eagle's Medium (Sigma Cat. No. D6546) with 10\% fetal bovine serum (Gibco ${ }^{\mathrm{TM}}$ cat\#10082147) and $100 \mathrm{U} / \mathrm{mL}$ penicillin/streptomycin (Gibco ${ }^{\mathrm{TM}}$ cat\#15140148). For subculture, Accutase ${ }^{\circledR}$ (Sigma, cat\#A6964) was used for a maximum of 10 passages.

\subsection{Experimental procedures}

\subsubsection{Immunocytochemistry}

\section{Primary Neurons:}

Cells were fixed for 10 min in $4 \%$ formaldehyde, washed and then stored in PBS until staining. Staining starts with permeabilizing the cells in $0.1 \%$ Triton for $10 \mathrm{~min}$. Blocking of nonspecific antibody binding sites was performed using $10 \%$ donkey serum in PBS for $1 \mathrm{~h}$ before primary antibody solution was 
added. Staining with DAPI was done to visualize nuclei of cells, Phalloidin was chosen for staining of actin filament to reveal the dendritic tree. Cells were incubated at $4^{\circ} \mathrm{C}$ overnight. Secondary antibodies were administered the next day after 3 washings with PBS and left on shaker without light exposure for 1 $\mathrm{h}$ at RT. Coverslips were mounted on glass slides using a drop ( $5 \mu \mathrm{l})$ of Prolong Gold (Thermo Fisher Scientific) after which slides were left at RT to harden overnight before imaging.

\section{IMG Cells:}

IMG cells plated on sterile glass coverslips and grown to $80 \%$ confluency, were fixed for 10 min in $4 \%$ formaldehyde and washed three times with PBS until staining. To begin immunochemical staining, cells were simultaneous blocked and permeabilized for one hour with PBS containing $3 \%$ bovine serum albumin (Sigma, cat\# A7030) and 0.1\% saponin (Sigma, cat\#47036) (microglia staining buffer). The primary antibody goat anti-lba-1 (Abcam, cat\#ab5076, 1:200 dilution) was applied for one hour at room temperature and followed by three, five-minute washes in $0.1 \%$ saponin in PBS. Addition of Phalloidin (Invitrogen cat\# A12379, 1:800 dilution) (conjugated to AlexaFluor-488) antibody along with donkey antigoat AF-555 (Abcam, cat\# a32816) secondary antibodies were incubated simultaneously to label the actin filaments and the primary anti-lba-1 antibody ( $1 \mathrm{~h}$ at RT in microglial staining buffer). Iba- 1 is a classic microglial marker ${ }^{44}$. Cells were mounted on glass slides using a drop $(\sim 5 \mu)$ of Prolong Gold with DAPI (Thermo Fisher Scientific) for nucleus visualization and left to harden overnight before imaging.

\subsubsection{Synapse marker Time curve}

Psilocybin solution (Merck \& Co, USA) was diluted in Neurobasal media to a final concentration of $1 \times 10^{-7}$ M. The dose was chosen based on confirmatory experiments from previous results by Ly et al. (2018). Due to the manufacturer's dilution of psilocybin in 50\% acetonitrile: $50 \%$ distilled water, a vehicle control with the corresponding concentration of acetonitrile (Sigma-Aldrich, Germany) was used. For insight into the impact of psilocybin on synaptic plasticity in hippocampal cells, the cells were initially studied at 4 time-points following administration of the substance ( $5 \mathrm{~min}, 15 \mathrm{~min}, 30 \mathrm{~min}, 1 \mathrm{~h}$ ). Cells were simultaneously harvested at DIV17 after fixation in $4 \%$ formaldehyde at room temperature $\left(21^{\circ} \mathrm{C}\right)$ for 10 min. Cells were stained with DAPI for nucleus visualization and the actin stain Alexa Fluor $^{\text {TM }} 488$ Phalloidin (Invitrogen cat\# A12379, 1:3000) was used to visualize dendrites and spines. Antibodies against presynaptic (guinea pig anti-Piccolo, SynapticSystems cat\#142104, 1:400) and postsynaptic (rabbit anti-Homer1, SynapticSystems cat\#160003, 1:400) proteins were used to assess plasticity inducing effects of psilocybin. Cell cultures were investigated at four additional timepoints after either psilocybin or vehicle treatment $(1,3,6$, and $24 \mathrm{~h})$. To verify and complement these time curves, a rabbit anti-Synapsin-1 antibody (Abcam cat\#ab64581, 1:400) was used to identify presynaptic nerve terminals in hippocampal cell cultures at eleven timepoints (5, 15, and 30 minutes, 1, 2, 3, 4, 5, 6, 24, and 72 hours).

\subsubsection{Imaging and analysis}

For synaptic markers, Airyscan imaging was applied (Zeiss LSM800-Airy 63x oil, NA 1.4 objective or Zeiss LSM900-Airy 63x NA 1.4 objective). Images were taken using optimized settings for the Airyscan and processed using the Zeiss Airyscan module. Second order dendrites from ten individual cells per well 
were imaged and analyzed. To quantify synaptic puncta, we used SynQuant ${ }^{45}$. To optimize the settings for SynQuant we randomly chose 10 images distributed between the different treatments. Then, we tested different settings to see when SynQuant detected most of the spines in all images without finding erroneous spines. This allowed us to find the settings that best detected the number of presynaptic, postsynaptic and colocalized puncta, respectively. We also tested other settings and got similar results; hence the program is robust when analyzing our data. SynQuant was also used to estimate size and intensity of the fluorescent puncta. The investigators performing imaging and analyses were blinded to treatment groups until the data were analyzed. IMG cells were imaged using a Zeiss LSM-710 confocal microscope at 20x magnification.

\subsubsection{Inflammation assay, TNF- $a$ and IL-6 quantification}

IMG cells were grown to $80 \%$ confluency in a 24-well plate, with approximately 50,000 cells initially seeded in each well. One hour prior to lipopolysaccharide (LPS) $(10 \mathrm{ng} / \mathrm{ml})$ (Sigma, cat\# L2880) addition, a range of concentrations of psilocybin were added to the cells $\left(10^{-5}, 10^{-6}, 10^{-7}, 10^{-8}\right.$, and $\left.10^{-9} \mathrm{M}\right)(\mathrm{n}=$ 3 biological replicates for each group). A vehicle control (acetonitrile) was also initially applied prior to LPS addition. After preincubation, cells were treated with $10 \mathrm{ng} / \mathrm{mL}$ LPS for $15 \mathrm{~h}$. Media was collected and cell viability was assessed using the CellTiter $96 \AA$ Aqueous One Solution Cell Proliferation Assay kit (MTS) (Promega, Madison, WI, cat\#G3580), which measures the formazan product that is produced in proportion to viable cell populations (measured using absorbance at $490 \mathrm{~nm}$ ). Mouse TNF- $\mathrm{a}$ and Interleukin-6 (IL-6) levels were measured independently (BioLegend's ELISA MAX ${ }^{\mathrm{TM}}$ Deluxe Sets, cat\#430904 and cat\#431304) according to the manufacturer's recommendations. Media from each biological replicate was measured in triplicate for TNF-a and IL-6 content.

\subsubsection{Statistics}

Synaptic puncta data were analyzed using a generalized linear model (GLM) followed by post-hoc analysis using the Holm method, comparing all treatments to the control group. The reason for using GLM was due to the variance differing considerably between the groups, making ANOVA unsuitable. Statistical calculations and graphical illustrations were performed in R and RStudio version 1.145646,47. For the inflammatory assay, the GLM was followed by post-hoc tests using the multicomp package, comparing all treatment groups to LPS alone and used the Holm method to adjust for multiple comparisons. P-values are reported in the text. Stars in the figures indicate level of significance : $p<=.1$ *: $p<=.05, * *: p<=.01, * \star *: p<=.001, * \star \star *: p<=.0001$.

\section{Results}

3.1 Psilocybin rapidly upregulates pre- and post-synaptic proteins Piccolo and Homer1 in primary mouse hippocampal neuronal cultures

To analyze the synaptogenic potential of psilocybin, we treated hippocampal neuronal cultures were stained with a pre-synaptic marker Piccolo, a protein important for synaptic vesicle retrieval ${ }^{48}$ and a post- 
synaptic marker, Homer1, a post-synaptic density scaffolding protein ${ }^{49}$. This allowed us to assess both pre-and post-synaptic changes in plasticity induction. Importantly, this also lets us quantify the number of locations where pre- and post-synaptic puncta co-localize.

We used SynQuant to identify and quantify synaptic puncta in hippocampal neurons and found robust responses to psilocybin treatment. Treatment with psilocybin significantly increased the number of presynaptic Piccolo (Fig. 2a) and post-synaptic Homer1 (Fig. 2b) puncta/ $\mu$ m at 1 ( $p=.000049$ and $p=.0053$ respectively) and $3 \mathrm{~h}(p=.000049$ and $p=0.014$ respectively) post-treatment. Interestingly, the density of both Piccolo and Homer 1 dropped to below baseline at $6 \mathrm{~h}(p=.046$ and $p=.0000058)$ before stabilizing at $24 \mathrm{~h}$.

The higher density of Piccolo than Homer1 puncta can appear puzzling at first. However, neurons in culture form very extensive axonal arborizations with many pre-synaptic enlargements, of which not all make contact with a dendrite. These regions can be functional and are considered "orphan" presynaptic sites (Krueger et al., 2003). It is therefore also important to look at the number of puncta where Piccolo and Homer1 co-localize, indicating presence of both pre- and post-synaptic machinery in the same region.

Similar to the increases of individual puncta, we found an increase in the number of colocalized markers (Fig. 2c, $1 \mathrm{~h} \mathrm{p}=.0000015,3 \mathrm{~h} \mathrm{p}=.0000016$ ). Next, we examined the intensity of the synaptic puncta and observed significant increases in Piccolo (Fig. 2d. $1 \mathrm{~h} \mathrm{p}=.00000766,3 \mathrm{~h} \mathrm{p}=.000000092,6 \mathrm{~h} \mathrm{p}$ $=.00061095$ ) and Homer1 (Fig. 2e. $1 \mathrm{~h} \mathrm{p}=.000008698,3 \mathrm{~h} \mathrm{p}=.000007855,6 \mathrm{~h} \mathrm{p}=.000008931$ ) intensity at 1 and $3 \mathrm{~h}$ post-psilocybin treatment, followed by a reduction at $6 \mathrm{~h}$. For both Piccolo and Homer 1 , the expression levels per puncta normalized to baseline at the $24 \mathrm{~h}$ timepoint. Previous studies have correlated increased synaptic protein fluorescence to increased functional synapses ${ }^{50}$ and increased post-synaptic secretory protein pathways ${ }^{51}$. One possible confounding factor could be discrepancies in detection of puncta with high protein expression compared to dimmer puncta. To investigate this, we made a density plot (Fig. 2f) to observe how intensity of Homer1 puncta affects spine detection. We found that in all the groups there were a large number of faint particles, regardless of the average intensity, indicating that SynQuant manages to detect both low and high intensity objects. Interestingly, the $1 \mathrm{~h}$ treatment group had mostly brighter puncta while the $3 \mathrm{~h}$ group had a high and low intensity group, possibly indicating that neurons were transitioning back to baseline, or that only some of the neurons were able to maintain the potentiation induced by psilocybin. In Fig. 2g, Airyscan images illustrate the distribution of synaptic markers on second order dendrites without (control) and with postpsilocybin treatment $1 \mathrm{~h}, 3 \mathrm{~h}$, and $6 \mathrm{~h}$ post-treatment.

\subsection{Biphasic regulation of presynaptic Synapsin-1 by psilocybin in primary mouse hippocampal neuronal cultures}

From the Piccolo and Homer1 data, we found that a dramatic increase in the number of pre- and postsynaptic sites occurred as early as $1 \mathrm{~h}$ after psilocybin treatment with a dramatic decrease at the $6 \mathrm{~h}$ timepoint. Hence, we sought to understand if these synaptic changes may occur even earlier and 
determine whether the dynamic activity ends or changes after the $24 \mathrm{~h}$ timepoint. To investigate this and to attempt to reproduce our findings with another presynaptic marker, we added timepoints at both ends of the time curve and used the anti-Synapsin- 1 antibody for our second analysis. Doing this, we observe gradual increases in Synapsin-1 density after only 15 minutes of psilocybin exposure. Similar to our prior experiment in Fig. 2, this increase peaked at $3 \mathrm{~h}$, with the density also dropping after the peak, albeit at an earlier timepoint, $4 \mathrm{~h}$, (Fig. 3a). However, at $72 \mathrm{~h}$ post-psilocybin treatment, a second significant increase in total number of Synapsin-1 puncta occurred. Similar trends in puncta intensity were observed during this time course as the previous, with increased signal mirroring increased puncta density (Fig. 3a). Interestingly, at the $72 \mathrm{~h}$ timepoint where the most puncta were observed, we did not observe increased intensity (Fig. 3b), perhaps indicating less functional/active ${ }^{51}$ or dormant synapses ${ }^{52}$. Representative Airyscan images of dendrites at $3 \mathrm{~h}, 72 \mathrm{~h}$ and controls are illustrated in Fig. 3c.

\subsection{Inversed effect of psilocybin on primary mouse cortical neuron cultures}

To determine if there is a regional-specific effect, we used primary neuronal cultures derived from mouse cortical brain tissue and again monitored Piccolo and Homer1 expression, focusing on $30 \mathrm{~min}, 1 \mathrm{~h}$, and 3 h timepoints. For Homer1, there was a decreasing trend in synaptic density (Fig. 4a), but no specific timepoint differed significantly from controls. The number of colocalized Piccolo and Homer1 puncta were decreased at both $1 \mathrm{~h}(\mathrm{p}=.012)$ and $3 \mathrm{~h}(\mathrm{p}=.0068)$ (Fig. 4c). Surprisingly, the number of Piccolo positive puncta decreased significantly at $1 \mathrm{~h}(\mathrm{p}=0.00157)$ and $3 \mathrm{~h}(\mathrm{p}=.000037)$ the duration of treatment (Fig. 4d). Strikingly, the intensity (Fig. $4 b$ \& e) or size of the puncta were not affected. Density and intensity of Homer1 puncta in cortical neurons (Fig. 4f) are homogenously spread across all treatment groups, with increased densities of lower intensity puncta at $30 \mathrm{~min}$ and $3 \mathrm{~h}$ post-psilocybin treatment. There are higher densities of low intensity puncta in the control group, with psilocybin treatments increasing the density of higher intensity Homer1 puncta. Representative images of synaptic markers a on second order dendrites without and with post-psilocybin treatment at different timepoints post-treatment are visualized via Airyscan images (Fig. 4g).

\subsection{Psilocybin is anti-inflammatory in immortalized microglia challenged with LPS}

We next sought to assess if psilocybin could affect cellular response to an inflammatory challenge. To test this, we used an immortalized mouse microglial cell line, which express the canonical microglial marker Iba-1 (Fig. 5a) and have previously been used as a microglial model system ${ }^{43}$. The cells were preincubated with various concentrations of psilocybin for $1 \mathrm{~h}$ followed by treatment with $10 \mathrm{ng} / \mathrm{mL}$ LPS. Compared to vehicle control, addition of LPS resulted in a clear increase of TNF-a (Fig. 5b). With increased doses of psilocybin against the LPS challenge, a trend in TNF-a decrease was observed at a dose of $10^{-7} \mathrm{M}$ with significant decreases observed at the $10^{-5} \mathrm{M}$ dose ( $\mathrm{p}=0.00035$, Fig. 5b). MTS assay results show mitogenic effects (increased viable population of cells) of LPS, which has been previously described ${ }^{53}$. The highest dose of psilocybin used in our assay $\left(10^{-5} \mathrm{M}\right)$ did not affect viability compared 
to the vehicle control, nor did it affect viability in combination with LPS (Fig. 5b). TNF-a levels begin a trend of decrease in LPS challenged IMG cells at a psilocybin dose of $10^{-7} \mathrm{M}$, with significant reductions reached at a dose of $10^{-5} \mathrm{M}(* *=\mathrm{p}=.00036)$ compared to LPS alone (Fig. $\left.5 \mathrm{c}\right)$. A trend for decreasing IL-6 levels released into media was observed (Fig. $5 \mathrm{~d}$ ), but significant decrease.

\section{Discussion}

Neuronal activity is known to rearrange and affect the function of neuronal circuits in the brain ${ }^{54}$. Plasticity promoting capacity is associated with upregulation of neurotrophic factors, such as brainderived neurotrophic factor (BDNF) ${ }^{5}$, and downregulation of plasticity opposing signaling, epitomized by the Nogo signaling system ${ }^{55,56}$. In addition, plasticity is affected by modulatory neurotransmitters such as serotonin ${ }^{57}$. Psychedelic compounds such as psilocybin, lysergic acid diethylamide (LSD), mescaline, 4-iodo-2,5-dimethoxyphenylisopropylamine (DOI) and N, N-dimethyltryptamine (DMT) have individual pharmacological properties, but all involve signaling through serotonergic receptors ${ }^{58}$. LSD has been shown to increase levels of immediate early genes Arc and c-Fos fivefold and twofold, respectively, in prefrontal cortex, with c-Fos mRNA expression additionally showing twofold increases in hippocampus and midbrain ${ }^{59}$. DOI stimulation of serotonergic receptors affects BDNF mRNA expression in a regionspecific manner, with higher doses producing a robust increase in parietal cortex and decrease in the dentate gyrus ${ }^{60}$. A number of psychedelic compounds, the most robust being LSD, were shown to promote neuritogenesis, spinogenesis, and synaptogenesis in cultured cortical neurons ${ }^{9}$. Taken together, these observations provide strong evidence that psychedelic compounds have plasticity evoking effects; however, little is known regarding the effects of psilocybin on synaptic activity. As interest in the psilocybin's therapeutic value for neurologically related diseases increases ${ }^{20,61}$, it has become important to investigate its cell-type specific actions of psilocybin in addition to its plasticity enhancing properties at the synaptic level.

To deepen our understanding of the impact of psilocybin on synaptic and structural plasticity we used hippocampal and cortical neuronal cell cultures to obtain time curves of the expression of number and intensity of structural synaptic proteins in response to psilocybin. Our results show that psilocybin rapidly and differentially affects pre- and postsynaptic protein puncta in a time- and region dependent manner. In hippocampal neuronal cultures, psilocybin rapidly increased the number of pre- and post-synaptic (Piccolo and Homer1) structures, peaking between 1 and $3 \mathrm{~h}$, with normalization back to baseline levels at $6 \mathrm{~h}$ post-treatment. The number and intensity of presynaptic protein, Synapsin-1, was assessed at shorter and longer time course and the highest number of puncta was found $72 \mathrm{~h}$ post psilocybin treatment, indicating an even more prolonged effect of the drug. The rapid peak of plasticity might be a productive window to place more focus on. Psilocybin is currently investigated as an adjuvant therapy to other modes of treatment, and by finding the optimal timing between psilocybin treatment, and for instance therapy, the effect might be even larger. Increased fluorescent intensity levels of synaptic puncta expression have previously been correlated to increased levels of functional synapses ${ }^{50}$ as well as post- 
synaptic machinery necessary for active synapses ${ }^{51}$. Intensity levels of Homer1, Piccolo, and Synapsin-1 puncta mirror the increased numbers of puncta we observe, with the exception of $72 \mathrm{~h}$ post-psilocybin treatment for Synapsin-1. At this timepoint, Synapsin-1 puncta reached the highest levels, though the intensity of these puncta was not increased. This may be a reflection of synapses becoming dormant, which is a reversible phenomenon ${ }^{52}$ (Crawford and Mennerick, 2012). Whether or not other synaptic proteins exhibit similar expression patterns at the $72 \mathrm{~h}$ timepoint will need to be further explored.

Interestingly, psilocybin treatment of cortical neuronal cell cultures did not change post-synaptic puncta counts (Homer1) but resulted in both decreased pre-synaptic puncta counts (Piccolo) and colocalization of pre- and post-synaptic sites. Intensity levels of puncta expression did not change over the course of the psilocybin treatment in cortical neuronal cultures. A recent study of effects of psilocybin in rats in vivo reports transcriptional up- and down-regulation of genes related to plasticity, specifically expression of Nr4a1, PSD95, Sgk1, ArC, $K B a$, and $E g r 2^{62}$. Our findings in the cortical cell culture match results by Jefsen et al. (2021) showing no changes in Synapsin-1 or Homer1 mRNA expression in the prefrontal cortex of rats; however, our results diverge from their rat hippocampal data as they did not find Synapsin-1 or Homer1 mRNA expression level changes. This is unlikely to be a species-specific difference but may be due to the timing of sacrifice after psilocybin treatment (90 minutes).

Overall, our findings support recent observations that psilocybin has a potent effect on synaptic density, as shown from prior studies showing increased expression levels of synaptic vesicle protein 2a (SV2A) in pigs following a single dose of psilocybin. The increases in the presynaptic SV2A protein were significantly increased 7 days post administration in both hippocampus and prefrontal cortex ${ }^{63}$. Shao et al., (2021) recently utilized a mouse model of learned helplessness (depression) and showed that a single dose of psilocybin ameliorated behavioral deficits ${ }^{64}$. They also found the single dose to significantly increase dendritic spine size and density in the medial frontal cortex a day after psilocybin treatment and persisting up to a month. While rapid effects of psilocybin have been less tested, our studies now provide a context for more short-term changes in synaptic architecture. In cortical neurons, we show that psilocybin has a rapid effect on the pre-synaptic side, resulting in downregulation of Piccolo within the first 3 hours, while having relatively little effect on the post synaptic side. This might appear paradoxical, but serotonin has been proposed to function as an expectation error signal ${ }^{65}$. We hypothesize that psilocybin might increase plasticity by removing potentially erroneous synapses before new synapses can be formed.

Psilocin is an unspecific serotonergic agonist and individual serotonergic receptors respond to different concentrations of psilocybin. High affinity is associated with the 5-HT7, 5-HT1D, and the 5-HT2A,B, and C receptors, while there is minimal interaction with the $5-\mathrm{HT} 3$ receptor $^{11}$. Mouse neurons express mRNA most for all of these receptors (minimally for $5 \mathrm{HT} 2 \mathrm{~B})^{66}$, while microglia most highly express mRNA for the $5-\mathrm{HT} 2 \mathrm{~B}$ and $\mathrm{C}$ receptors ${ }^{67}$. As the concentration of psilocybin and its active metabolite psilocin reasonably differs over time due to metabolism in our cell cultures, activation of various serotonergic receptors, inhibitory as well as excitatory ${ }^{68}$, are likely occurring over the timescales in the present study. 
Further studies are needed to understand which concentration of psilocybin optimally heightens plasticity. Access to an increased level of plasticity could potentially serve as a primary mechanism for the therapeutic value of psilocybin. Furthermore, by knowing when plasticity is maximal after psilocybin treatment may allow other therapeutic interventions, e.g., post stroke rehabilitation. to be timed to these windows.

In a recent study using pigs ${ }^{69}$, a single dose of psilocybin was shown to significantly upregulate many immunological gene pathways in the PFC, as determined by RNA sequencing and gene set enrichment analysis (GSEA). The most enriched pathways included GO Immune Response, GO Regulation of Immune Response, GO Immune Effector Process, and GO Innate Immune Response, among others related to response to interferon proteins and cytokines. Although these results point to a clear immunologic effect of a single psilocybin administration, the research group was unable to verify several candidate genes using RTqPCR. Though no inflammatory challenge was applied in this study, these results clearly show a relationship between the brain's immune response and psilocybin. Another recent study demonstrated immunomodulatory effects (morphological and protein expression changes) in primary microglia treated with DMT or psilocin. This study provides further evidence of direct action of serotonergic psychedelics on microglia ${ }^{70}$.

Our study expands understanding of the anti-inflammatory capacity of psychedelics affecting the serotonergic system ${ }^{29,30}$. We used an immortalized microglial (IMG) cell line, previously characterized to recapitulate key features of primary microglial activation ${ }^{43}$, challenged with LPS as a model of neuroinflammation. We demonstrate a significantly reduced release of the canonical inflammatory protein TNF-a by IMG cells pretreated with a $10^{-5} \mathrm{M}$ psilocybin and subjected to an LPS challenge. The pleiotropic cytokine IL-6, possessing both pro- and anti-inflammatory properties, usually accompanies TNF-a in inflammation states ${ }^{71}$. Hence, we also examined its presence in cell culture media following an LPS challenge. Although IL-6 presence trended down at a high dose of psilocybin, this cytokine was not significantly reduced.

While studies associated with psilocybin's anti-inflammatory effects are limited due to its rapid metabolic breakdown, an inflammation suppressing pharmacophore of other psychedelics which activate the 5HT2A receptor was recently described using a rat model of asthma ${ }^{29}$. This study showed significant reductions of expression of inflammation-related genes in the lung, including TNF- $a$ and other cytokines. Interestingly, a recent study showed significant increases in mRNA expression of $k B a$ (nuclear factor kappa light-chain-enhancer polypeptide gene enhancer of B-cells inhibitor, alpha) and Dusp1 (dualspecificity-phosphate 1) in hippocampus and PFC of a rat after single administrations of different doses of psilocybin ${ }^{62}$. Increases in protein levels was confirmed in hippocampus but not in the PFC. IkBa is an endogenous inhibitor of the transcription factor NF-kB (nuclear factor kappa-light-chain-enhancer of activated $B$ cells $)^{72}$, which is inducible by LPS $^{73}$ and central to maximal production of TNF- $a^{74}$. Interestingly, lkBa has been shown to play an important function in synaptogenesis and might be a common target for synaptic and anti-inflammatory effects ${ }^{75}$. Dusp 1 has been previously shown to have 
potent anti-inflammatory effects. Dusp1 knock-out mice show 10-fold increases compared to wild type animals of several cytokines in blood serum, including TNF-a ${ }^{76}$. Importantly, Dusp1 promotes the formation of an anti-inflammatory phenotype in microglia ${ }^{77}$, with other studies further demonstrating similar effects ${ }^{78,79}$. Psilocybin's induction of $\mathrm{IkBa}$ and Dusp1 is a possible mechanism whereby the drug inhibits inflammation. Further studies are needed to confirm this.

Altogether, current evidence in the literature and our studies represents possibilities for new classes of drugs that act on serotonergic receptors with anti-inflammatory capacity. Allosteric targeting of these receptors to activate downstream anti-inflammatory pathways but avoid psychedelic effects may be a fruitful area of research in the future.

\section{Conclusions}

We investigated the effect of psilocybin on pre- and postsynaptic markers over time in cultured hippocampal neurons and found a biphasic increase of synaptic density and puncta intensity with peaks at $3 \mathrm{~h}$ and $72 \mathrm{~h}$. Additionally, we show decreased presynaptic protein expression in cortical neuronal cultures up to $3 \mathrm{~h}$ post psilocybin treatment. Overall, our primary neuronal cell culture models were effective for studying the synaptic alterations caused by psilocybin and provide concrete evidence for the plasticity-inducing effects of the drug. Lastly, we found an anti-inflammatory effect of psilocybin that significantly reduced the release of inflammatory proteins in a microglial cell line $15 \mathrm{~h}$ after an LPS challenge.

\section{Declarations}

\section{Author contributions}

GS*; Conceptualization; Methodology; Data Curation; Formal analysis; Funding Acquisition; Investigation; Project administration; Writing original draft; Writing - review \& editing. EG*; Conceptualization; Methodology; Data Curation; Formal analysis; Funding Acquisition; Investigation; Project administration; Writing - review \& editing. NK, Investigation, Validation. CPH Hjelle, Investigation. OH, Investigation. KW, Investigation. AB, Investigation. FvK, Methodology; Visualization, Review \& editing. LO, Funding Acquisition; Investigation; Project administration; Writing - review \& editing; Supervision; Resources. TK, Conceptualization; Methodology; Data Curation; Formal analysis; Funding Acquisition; Investigation; Project administration; Writing - review \& editing; Validation; Supervision. * Equal contributions.

\section{Acknowledgements}

The imaging was performed in the Biomedicum Imaging Core (BIC) with support from the Karolinska Institutet. This work was supported by the Swedish Research Council K2012-62X-03185-42-4 (LO), the Swedish Brain Foundation 2018 (LO), a donation by Per Nydahl 2019 (LO), the Karolinska Institute Clinical Scientist Training Programme (CSTP) 2013 (GS), the Karolinska Institutet Program for "Forskar AT" 2016 (GS), the Swedish Brain Foundation 2020 (GS), and the Intramural Research Program of the 
National Institute on Aging, National Institutes of Health, Baltimore, MD, United States (EG). EG is supported by the National Institutes of Health-Karolinska Institute Graduate Partnership Program. Graphical abstract created on BioRender.com with added artwork from Lauren Brick, Visual Media core, National Institute on Drug Abuse, National Institutes of Health, USA.

Competing interest

GS and FvK are co-founders of Amidea Sweden AB and FvK is the co-founder of Osmond Labs AB; two drug discovery companies. None of the work herein has been carried out in collaboration with these companies and neither GS or FvK, nor the companies or the Karolinska Institutet have been compensated financially. The remaining authors declare no competing interests.

Availability of materials and data

The datasets generated during and/or analysed during the current study are available from the corresponding author on reasonable request.

\section{References}

1. Yiu, G. \& He, Z. Glial inhibition of CNS axon regeneration. Nat Rev Neurosci 7, 617-627 (2006).

2. Akbik, F., Cafferty, W. B. J. \& Strittmatter, S. M. Myelin associated inhibitors: a link between injuryinduced and experience-dependent plasticity. Exp. Neurol. 235, 43-52 (2012).

3. Huebner, E. A. \& Strittmatter, S. M. Axon regeneration in the peripheral and central nervous systems. Results Probl Cell Differ 48, 339-351 (2009).

4. Schwab, M. E. \& Strittmatter, S. M. Nogo limits neural plasticity and recovery from injury. Curr. Opin. Neurobiol. 27, 53-60 (2014).

5. McAllister, A. K., Katz, L. C. \& Lo, D. C. Neurotrophins and Synaptic Plasticity. Annual Review of Neuroscience 22, 295-318 (1999).

6. Baloh, R. H., Enomoto, H., Johnson, E. M. \& Milbrandt, J. The GDNF family ligands and receptors implications for neural development. Current Opinion in Neurobiology 10, 103-110 (2000).

7. Berlucchi, G. \& Buchtel, H. A. Neuronal plasticity: historical roots and evolution of meaning. Exp Brain Res 192, 307-319 (2009).

8. McGaugh, J. L. Drug enhancement of memory consolidation: historical perspective and neurobiological implications | SpringerLink. https://link.springer.com/article/10.1007\%2Fs00213008-1285-6 (2008).

9. Ly, C. et al. Psychedelics Promote Structural and Functional Neural Plasticity. Cell Reports 23, 31703182 (2018).

10. Passie, T., Seifert, J., Schneider, U. \& Emrich, H. M. The pharmacology of psilocybin. Addiction Biology 7, 357-364 (2002). 
11. Halberstadt, A. L. \& Geyer, M. A. Multiple receptors contribute to the behavioral effects of indoleamine hallucinogens. Neuropharmacology 61, 364-381 (2011).

12. McKenna, D. J., Repke, D. B., Lo, L. \& Peroutka, S. J. Differential interactions of indolealkylamines with 5-hydroxytryptamine receptor subtypes. Neuropharmacology 29, 193-198 (1990).

13. Nichols, D. E. Hallucinogens. Pharmacology \& Therapeutics 101, 131-181 (2004).

14. Amsterdam, J. van, Opperhuizen, A. \& Brink, W. van den. Harm potential of magic mushroom use: A review. Regulatory Toxicology and Pharmacology 59, 423-429 (2011).

15. Brown, R. T. et al. Pharmacokinetics of Escalating Doses of Oral Psilocybin in Healthy Adults. Clin Pharmacokinet 56, 1543-1554 (2017).

16. Moreno, F. A., Wiegand, C. B., Taitano, E. K. \& Delgado, P. L. Safety, tolerability, and efficacy of psilocybin in 9 patients with obsessive-compulsive disorder. J Clin Psychiatry 67, 1735-1740 (2006).

17. Carhart-Harris, R. et al. Trial of Psilocybin versus Escitalopram for Depression. New England Journal of Medicine 384, 1402-1411 (2021).

18. Bogenschutz, M. P. et al. Psilocybin-assisted treatment for alcohol dependence: A proof-of-concept study. J Psychopharmacol 29, 289-299 (2015).

19. Johnson, M. W., Garcia-Romeu, A. \& Griffiths, R. R. Long-term Follow-up of Psilocybin-facilitated Smoking Cessation. Am J Drug Alcohol Abuse 43, 55-60 (2017).

20. Nichols, D. E., Johnson, M. W. \& Nichols, C. D. Psychedelics as Medicines: An Emerging New Paradigm. Clinical Pharmacology \& Therapeutics 101, 209-219 (2017).

21. Schindler, E. A. D. et al. Indoleamine Hallucinogens in Cluster Headache: Results of the Clusterbusters Medication Use Survey. Journal of Psychoactive Drugs 47, 372-381 (2015).

22. Sewell, R. A., Halpern, J. H. \& Pope, H. G. Response of cluster headache to psilocybin and LSD. Neurology 66, 1920-1922 (2006).

23. Schindler, E. A. D. et al. Exploratory Controlled Study of the Migraine-Suppressing Effects of Psilocybin. Neurotherapeutics (2020) doi:10.1007/s13311-020-00962-y.

24. Barrett, F. S., Doss, M. K., Sepeda, N. D., Pekar, J. J. \& Griffiths, R. R. Emotions and brain function are altered up to one month after a single high dose of psilocybin. Scientific Reports 10, 1-14 (2020).

25. Carhart-Harris, R. L. et al. Psilocybin with psychological support for treatment-resistant depression: an open-label feasibility study. The Lancet Psychiatry 3, 619-627 (2016).

26. Griffiths, R. R. et al. Psilocybin produces substantial and sustained decreases in depression and anxiety in patients with life-threatening cancer: A randomized double-blind trial. J Psychopharmacol 30, 1181-1197 (2016).

27. Madsen, M. K. et al. A single psilocybin dose is associated with long-term increased mindfulness, preceded by a proportional change in neocortical 5-HT2A receptor binding. European Neuropsychopharmacology 33, 71-80 (2020).

28. Catlow, B. J., Song, S., Paredes, D. A., Kirstein, C. L. \& Sanchez-Ramos, J. Effects of psilocybin on hippocampal neurogenesis and extinction of trace fear conditioning. Exp Brain Res 228, 481-491 
(2013).

29. Flanagan, T. W. et al. Structure-Activity Relationship Analysis of Psychedelics in a Rat Model of Asthma Reveals the Anti-Inflammatory Pharmacophore. ACS Pharmacol. Transl. Sci. 4, 488-502 (2021).

30. Flanagan, T. W. Psychedelics as anti-inflammatory agents. International Review of Psychiatry 30, 363-375 (2018).

31. Harry, G. J. \& Kraft, A. D. Neuroinflammation and Microglia: Considerations and approaches for neurotoxicity assessment. Expert Opin Drug Metab Toxicol 4, 1265-1277 (2008).

32. Nkadimeng, S. M., Nabatanzi, A., Steinmann, C. M. L. \& Eloff, J. N. Phytochemical, Cytotoxicity, Antioxidant and Anti-Inflammatory Effects of Psilocybe Natalensis Magic Mushroom. Plants 9, 1127 (2020).

33. Jung, Y. J. et al. Repurposing Immunomodulatory Imide Drugs (IMiDs) in Neuropsychiatric and Neurodegenerative Disorders. Front. Neurosci. 15, (2021).

34. Troubat, R. et al. Neuroinflammation and depression: A review. European Journal of Neuroscience 53, 151-171 (2021).

35. Dinis-Oliveira, R. J. Metabolism of psilocybin and psilocin: clinical and forensic toxicological relevance. Drug Metab Rev 49, 84-91 (2017).

36. Rambousek, L., Palenicek, T., Vales, K. \& Stuchlik, A. The Effect of Psilocin on Memory Acquisition, Retrieval, and Consolidation in the Rat. Frontiers in Behavioral Neuroscience 8, (2014).

37. Sakashita, Y. et al. Effect of Psilocin on Extracellular Dopamine and Serotonin Levels in the Mesoaccumbens and Mesocortical Pathway in Awake Rats. Biological and Pharmaceutical Bulletin 38, 134-138 (2015).

38. Truscott, R. J. W. \& Manthey, M. K. The oxidation of 4-hydroxyindole as a model system for the oxidation of 4-hydroxyindolyl-3-methyl glucosinolate. Journal of the Science of Food and Agriculture 47, 191-195 (1989).

39. Brun-Heath, I. et al. Differential expression of the bone and the liver tissue non-specific alkaline phosphatase isoforms in brain tissues. Cell Tissue Res 343, 521-536 (2011).

40. Horita, A. \& Weber, L. J. Dephosphorylation of psilocybin to psilocin by alkaline phosphatase. Proc Soc Exp Biol Med 106, 32-34 (1961).

41. Horita, A. \& Weber, L. J. The enzymic dephosphorylation and oxidation of psilocybin and psilocin by mammalian tissue homogenates. Biochem Pharmacol 7, 47-54 (1961).

42. Horita, A. Some biochemical studies on psilocybin and psilogin. J Neuropsychiatr 4, 270-273 (1963).

43. McCarthy, R. C. et al. Characterization of a novel adult murine immortalized microglial cell line and its activation by amyloid-beta. J Neuroinflammation 13, 21 (2016).

44. Korzhevskii, D. E. \& Kirik, O. V. Brain Microglia and Microglial Markers. Neuroscience and Behavioral Physiology 3, 284-290 (2016). 
45. Wang, Y. et al. SynQuant: an automatic tool to quantify synapses from microscopy images. Bioinformatics 36, 1599-1606 (2019).

46. R Core Team. R Core Team (2019). R: A language and environment for statistical computing. $R$ Foundation for Statistical Computing, Vienna, Austria. http://www.R-project.org/. (2019).

47. RStudio Team. RStudio: Integrated Development for R. RStudio, Inc., Boston, MA. RStudio Support http://www.rstudio.com/. (2015).

48. Ackermann, F. et al. Critical role for Piccolo in synaptic vesicle retrieval. Elife 8, (2019).

49. Jaubert, P. J. et al. Complex, multimodal behavioral profile of the Homer1 knockout mouse. Genes, Brain and Behavior 6, 141-154 (2007).

50. Shapira, M. et al. Unitary assembly of presynaptic active zones from Piccolo-Bassoon transport vesicles. Neuron 38, 237-252 (2003).

51. Gürth, C.-M., Dankovich, T. M., Rizzoli, S. O. \& D'Este, E. Synaptic activity and strength are reflected by changes in the post-synaptic secretory pathway. Sci Rep 10, 20576 (2020).

52. Crawford, D. C. \& Mennerick, S. Presynaptically Silent Synapses: Dormancy and Awakening of Presynaptic Vesicle Release. Neuroscientist 18, 216-223 (2012).

53. Pyo, H., Jou, I., Jung, S., Hong, S. \& Joe, E. H. Mitogen-activated protein kinases activated by lipopolysaccharide and beta-amyloid in cultured rat microglia. Neuroreport 9, 871-874 (1998).

54. Ganguly, K. \& Poo, M. Activity-Dependent Neural Plasticity from Bench to Bedside. Neuron 80, 729741 (2013).

55. Josephson, A. et al. Activity-induced and developmental downregulation of the Nogo receptor. Cell Tissue Res. 311, 333-342 (2003).

56. Karlén, A. et al. Nogo receptor 1 regulates formation of lasting memories. Proc. Natl. Acad. Sci. U.S.A. 106, 20476-20481 (2009).

57. Kraus, C., Castrén, E., Kasper, S. \& Lanzenberger, R. Serotonin and neuroplasticity - Links between molecular, functional and structural pathophysiology in depression. Neuroscience \& Biobehavioral Reviews 77, 317-326 (2017).

58. Nichols, D. E. Psychedelics. Pharmacol Rev 68, 264-355 (2016).

59. Nichols, C. D. \& Sanders-Bush, E. A Single Dose of Lysergic Acid Diethylamide Influences Gene Expression Patterns within the Mammalian Brain. Neuropsychopharmacology 26, 634-642 (2002).

60. Vaidya, V. A., Marek, G. J., Aghajanian, G. K. \& Duman, R. S. 5-HT2A Receptor-Mediated Regulation of Brain-Derived Neurotrophic Factor mRNA in the Hippocampus and the Neocortex. J Neurosci 17, 2785-2795 (1997).

61. Kozlowska, U., Nichols, C., Wiatr, K. \& Figiel, M. From Psychiatry to Neurology: Psychedelics as Prospective Therapeutics for Neurodegenerative Disorders. Journal of Neurochemistry n/a,.

62. Jefsen, O. H., Elfving, B., Wegener, G. \& Müller, H. K. Transcriptional regulation in the rat prefrontal cortex and hippocampus after a single administration of psilocybin. J Psychopharmacol 35, 483493 (2021). 
63. Raval, N. R. et al. A Single Dose of Psilocybin Increases Synaptic Density and Decreases 5-HT2A Receptor Density in the Pig Brain. Int J Mol Sci 22, (2021).

64. Shao, L.-X. et al. Psilocybin induces rapid and persistent growth of dendritic spines in frontal cortex in vivo. 2021.02.17.431629 https://www.biorxiv.org/content/10.1101

/2021.02.17.431629v1

(2021) doi:10.1101/2021.02.17.431629.

65. Matias, S., Lottem, E., Dugué, G. P. \& Mainen, Z. F. Activity patterns of serotonin neurons underlying cognitive flexibility. eLife 6, e20552 (2017).

66. Zhang, Y. et al. An RNA-Sequencing Transcriptome and Splicing Database of Glia, Neurons, and Vascular Cells of the Cerebral Cortex. J Neurosci 34, 11929-11947 (2014).

67. Bennett, M. L. et al. New tools for studying microglia in the mouse and human CNS. Proc Natl Acad Sci U S A 113, E1738-E1746 (2016).

68. Berumen, L. C., Rodríguez, A., Miledi, R. \& García-Alcocer, G. Serotonin Receptors in Hippocampus. ScientificWorldJournal 2012, (2012).

69. Donovan, L. L. et al. Effects of a single dose of psilocybin on behaviour, brain 5-HT2A receptor occupancy and gene expression in the pig. European Neuropsychopharmacology 42, 1-11 (2021).

70. Kozlowska, U., Klimczak, A., Wiatr, K. \& Figiel, M. The DMT and Psilocin Treatment Changes CD11b + Activated Microglia Immunological Phenotype. bioRxiv 2021.03.07.434103, (2021).

71. Scheller, J., Chalaris, A., Schmidt-Arras, D. \& Rose-John, S. The pro- and anti-inflammatory properties of the cytokine interleukin-6. Biochim Biophys Acta 1813, 878-888 (2011).

72. Baeuerle, P. A. \& Baltimore, D. IkB: a Specific Inhibitor of the NF-kB Transcription Factor. Science 242, $540-546$ (1988).

73. Sen, R. \& Baltimore, D. Inducibility of K immunoglobulin enhancer-binding protein NF-kB by a posttranslational mechanism. Cell 47, 921-928 (1986).

74. Blackwell, T. S. \& Christman, J. W. The Role of Nuclear Factor- $\mathrm{k}$ B in Cytokine Gene Regulation. Am J Respir Cell Mol Biol 17, 3-9 (1997).

75. Alberini, C. M. \& Kandel, E. R. The Regulation of Transcription in Memory Consolidation. Cold Spring Harb Perspect Biol 7, (2015).

76. Hammer, M. et al. Increased inflammation and lethality of Dusp1-/- mice in polymicrobial peritonitis models. Immunology 131, 395-404 (2010).

77. Wang, X. et al. DUSP1 Promotes Microglial Polarization toward M2 Phenotype in the Medial Prefrontal Cortex of Neuropathic Pain Rats via Inhibition of MAPK Pathway. ACS Chem Neurosci 12, 966-978 (2021).

78. Abraham, S. M. et al. Antiinflammatory effects of dexamethasone are partly dependent on induction of dual specificity phosphatase 1. J Exp Med 203, 1883-1889 (2006).

79. Peng, H.-Z., Yun, Z., Wang, W. \& Ma, B.-A. Dual specificity phosphatase 1 has a protective role in osteoarthritis fibroblast-like synoviocytes via inhibition of the MAPK signaling pathway. Mol Med 


\section{Figures}

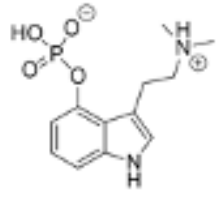

psilocybin

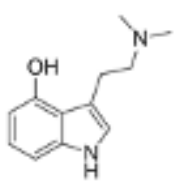

psilocin

\section{Figure 1}

Molecular structures for the naturally occurring compounds psilocybin and psilocin, found in mushrooms of the Psilocybe genus. Dephosphorylation of psilocybin to its CNS-active metabolite, psilocin, is readily achieved via the action of alkaline phosphatases ${ }^{42}$, expressed in living organisms as well as in cell cultures $^{39}$. Drawn with PerkinElmer ChemDraw 18.

a

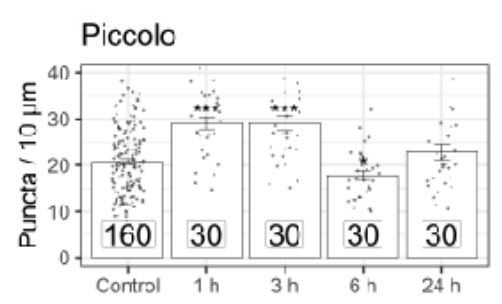

d

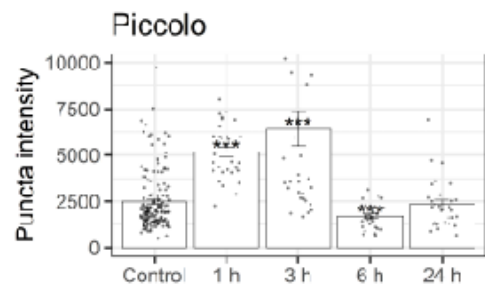

Control

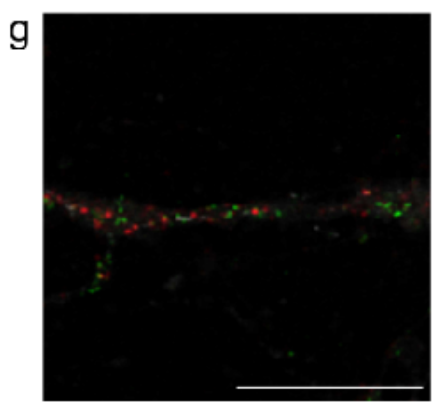

b

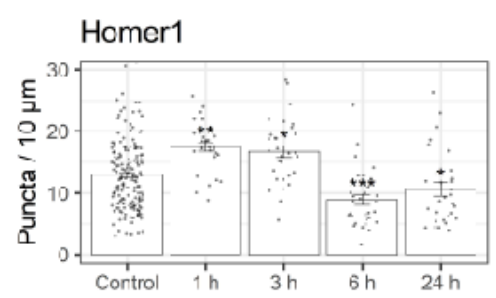

e

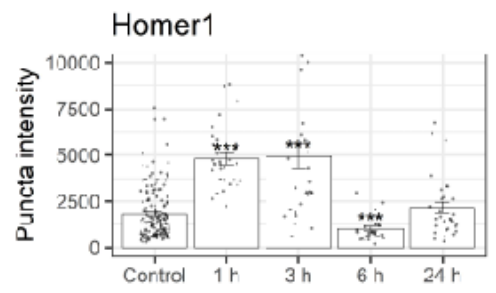

$1 \mathrm{~h}$

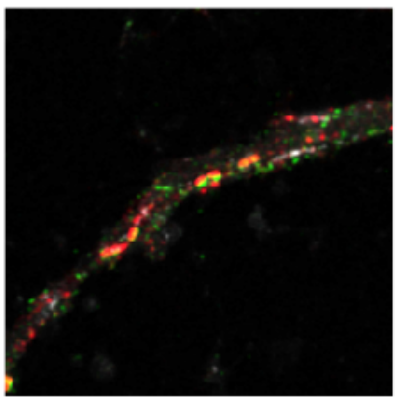

C

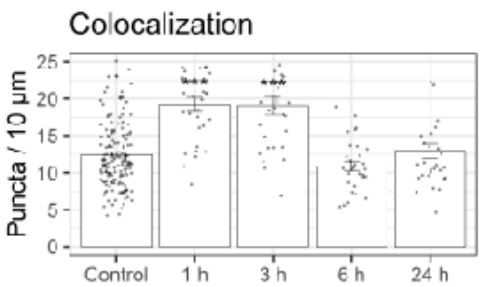

f

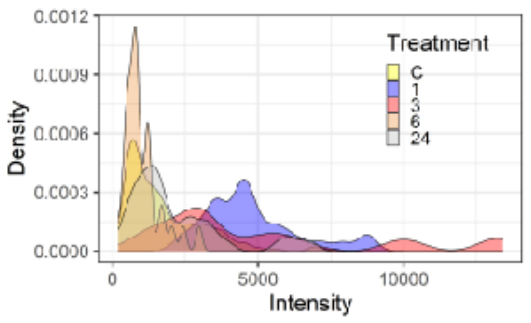

$6 \mathrm{~h}$
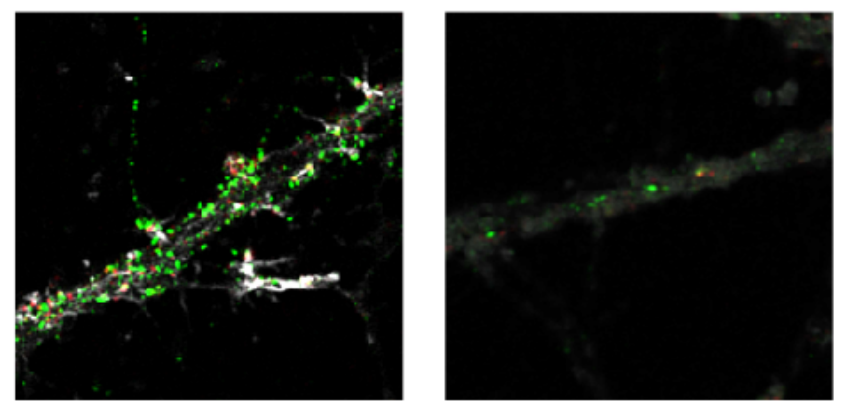

Figure 2 
Psilocybin rapidly and transiently increases the number of synaptic puncta. a) Number of pre-synaptic Piccolo puncta per $10 \mu \mathrm{m}$ as a marker of pre-synaptic density ( $n=$ number of neurons per group, valid for b-f, $c=$ controls). B) The number of Homer1 puncta as a marker of post-synaptic density and (c) the number of puncta with co-localization of Piccolo and Homer1 as markers of functional synaptic density. The mean intensity of the synaptic puncta is shown in (d) for piccolo and $\odot(e)$ for Homer1. (f) shows a density plot of the intensity of Homer1. (g) Airyscan images visualizing the distribution of synaptic markers on second order dendrites in cultured hippocampal neurons without (control) and with postpsilocybin treatment $1 \mathrm{~h}, 3 \mathrm{~h}$, and $6 \mathrm{~h}$ post-treatment. Green: presynaptic Piccolo, red: postsynaptic Homer1, scale bar $=10 \mu \mathrm{m}$. All treatment groups are compared to the control group. Error bars $=\mathrm{SEM}$.

a

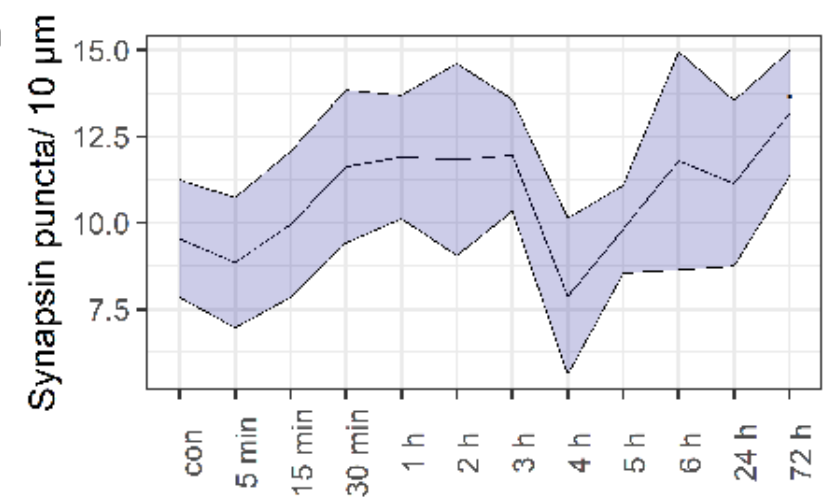

Control

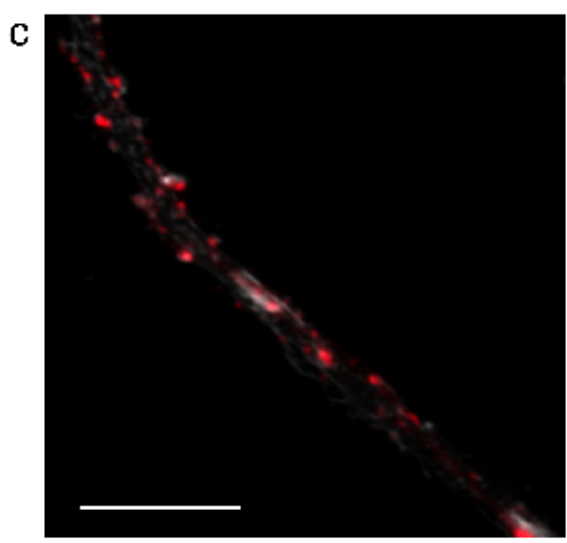

b

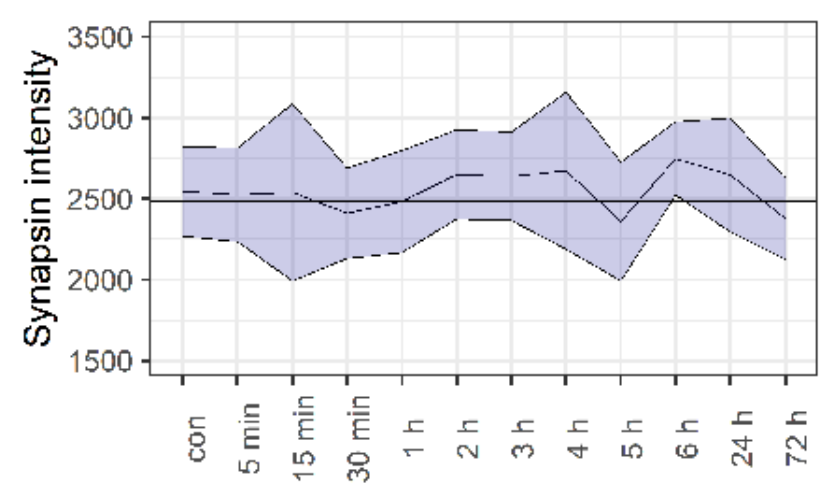

$3 \mathrm{~h}$

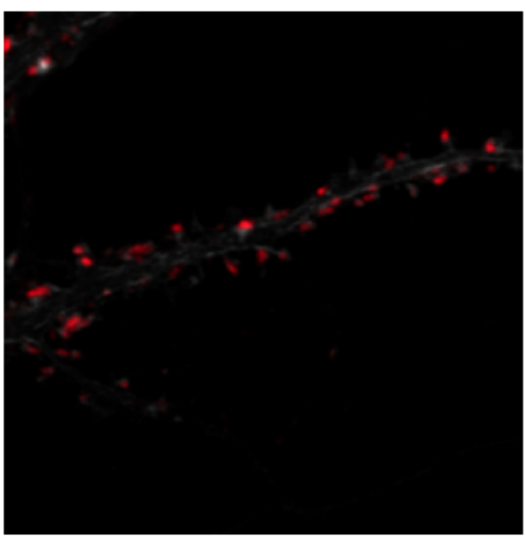

$72 \mathrm{~h}$

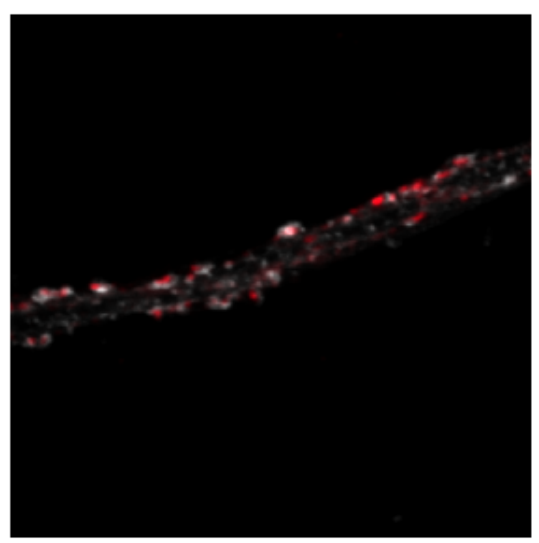

\section{Figure 3}

Bi-phasic changes in plasticity during 3 days in vitro. (a) The number of Synapsin-1 positive puncta was quantified at 11 timepoints after psilocybin stimulation to assess the time course of pro-synaptogenic potential of psilocybin. (b) The mean intensity of each puncta was also assessed (b). The shaded area shows $95 \%$ confidence interval. (c) representative images of dendrites. Red $=$ Synapsin-1, white $=$ phalloidin, Scale bar $=10 \mu \mathrm{m}$. 
a

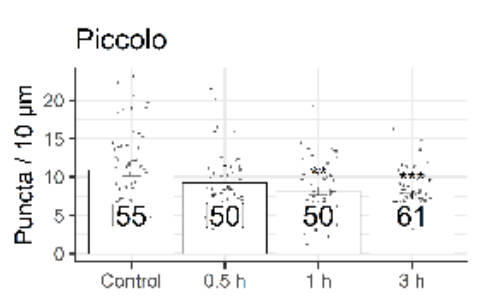

d

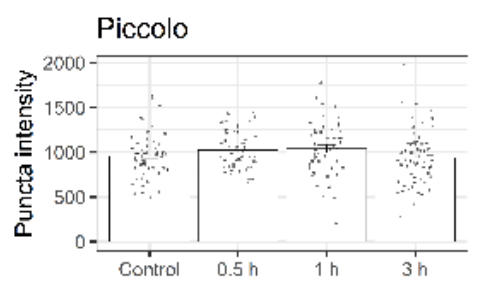

Control

g

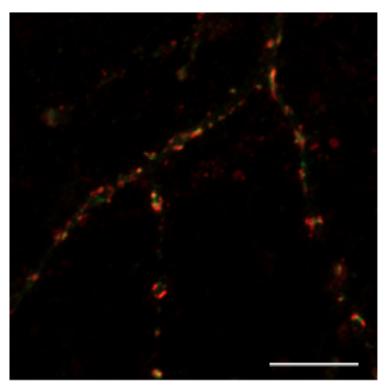

b

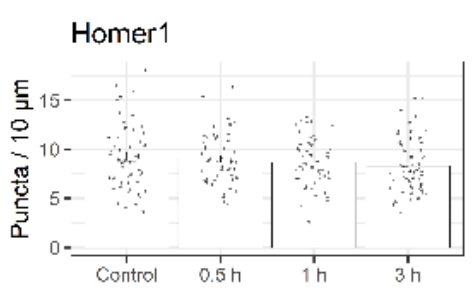

e

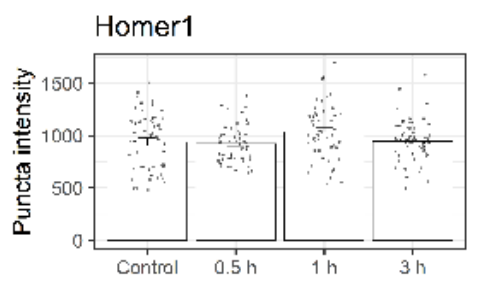

0.5

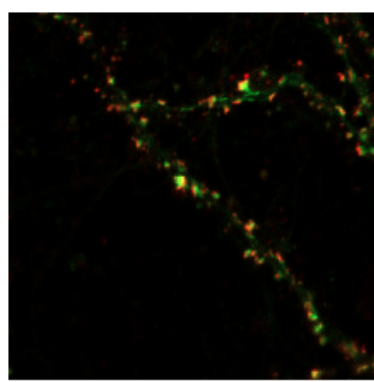

C

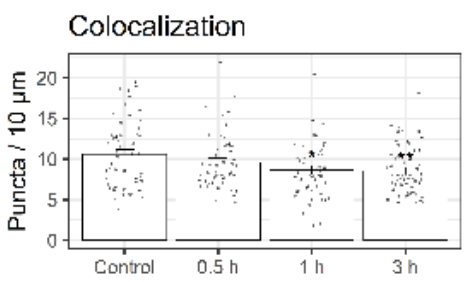

f

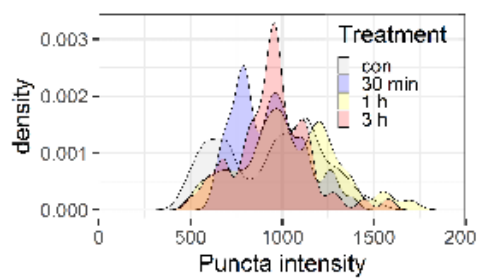

$3 \mathrm{~h}$
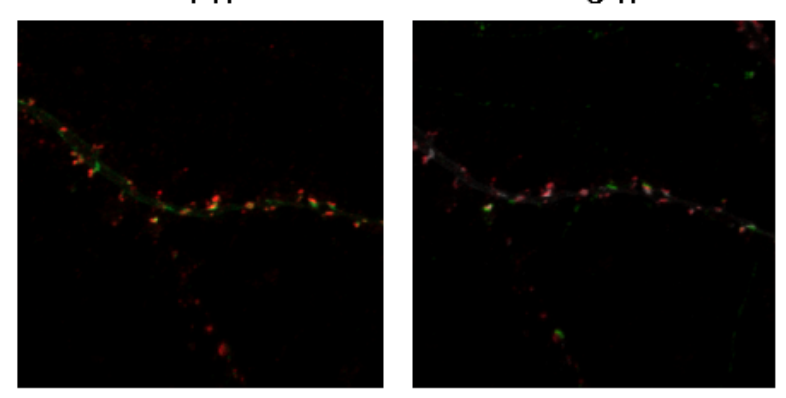

\section{Figure 4}

Psilocybin primarily affects the pre-synaptic terminals in cortical neurons. Cortical neurons were treated with psilocybin $3 \mathrm{~h}, 1 \mathrm{~h}$ or 30 min before fixation to assess pro-synaptogenic effects. (a) Piccolo positive puncta per $10 \mu \mathrm{m}$, (b) Homer1 positive puncta per $10 \mu \mathrm{m}$. (c) The number of Homer1 and Piccolo puncta that colocalized (d) mean puncta intensity of piccolo positive puncta (e) mean intensity of Homer 1 positive puncta, $(f)$ shows a density plot of the intensity of Homer1 and (g) Airyscan images visualizing the distribution of synaptic markers on second order dendrites in cultured cortical neurons without and with post-psilocybin treatment $1 \mathrm{~h}, 3 \mathrm{~h}$, and $6 \mathrm{~h}$ post-treatment. Green: presynaptic Piccolo, red: postsynaptic Homer1, scale bar= $10 \mu \mathrm{m}$. All treatment groups are compared to the control group. Error bars = SEM 
a

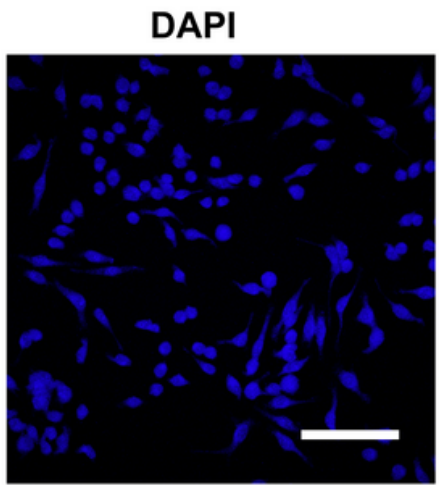

b

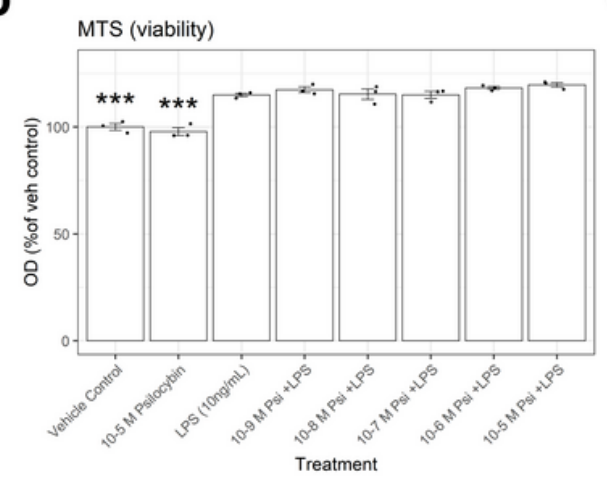

Phalloidin

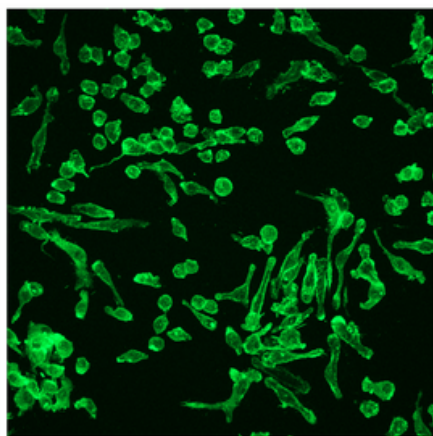

C

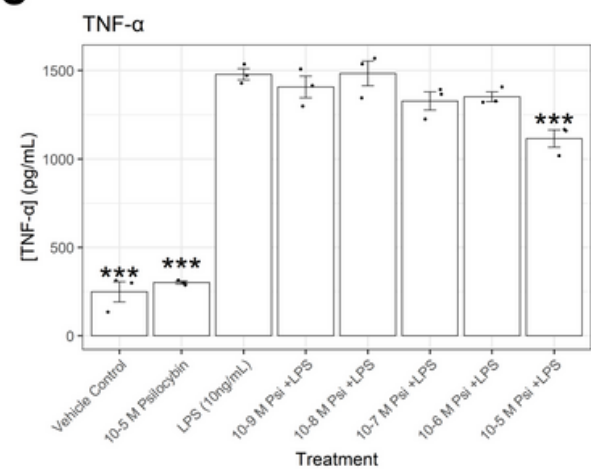

Iba1

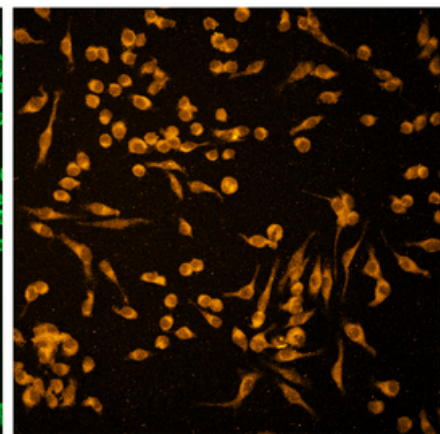

Merge

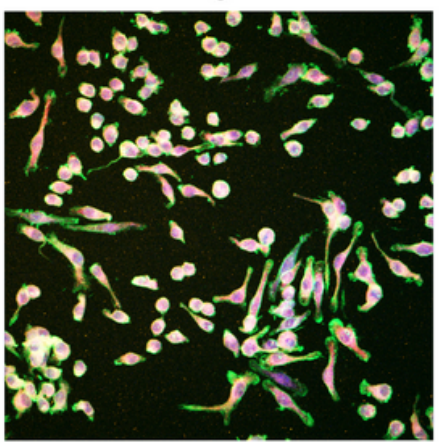

d

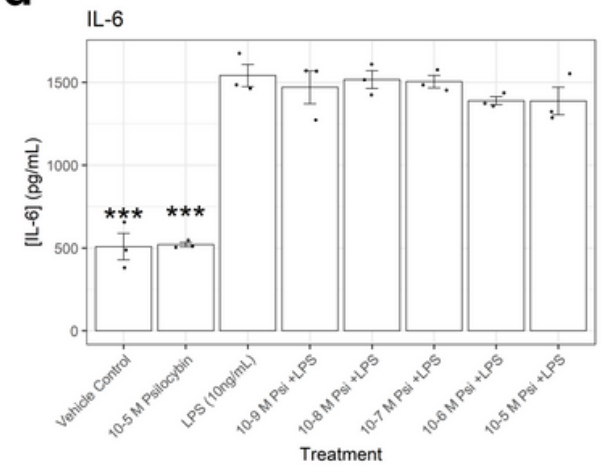

\section{Figure 5}

IMG cells used as a microglial model of neuroinflammation. a) IMG cells express the canonical microglial marker Iba-1. DAPI and Phalloidin are used to visualize nuclei and for morphological assessment. b) Following a $1 \mathrm{~h}$ preincubation with psilocybin and a $15 \mathrm{~h}$ coincubation with $10 \mathrm{ng} / \mathrm{mL}$ LPS, b) a strong mitogenic effect (increased viability) of LPS is observed across all treatment groups compared to vehicle control (

$=p<.0001)$. No differences between LPS + Psilocybin groups vs. LPS alone were observed and psilocybin alone had no effect on cell viability. c) TNF-a levels begin a trend of decrease in LPS challenged IMG cells at a psilocybin dose of 10-7 M, with significant reductions at a dose of 10-5 M (**=p=.0036) compared to LPS alone. d) Although there was a trend for decreasing IL-6 levels released into media, a significant decrease was not observed. (Error bars= SEM; Measure bar= 50mm). 